\title{
Christoffel words and the Calkin-Wilf tree
}

\author{
Alessandro De Luca \\ Dipartimento di Scienze Fisiche, Università degli Studi di Napoli Federico II \\ via Cintia, Monte S. Angelo - 80126 Napoli, Italy \\ Department of Mathematics, University of Turku \\ 20014 Turku, Finland \\ alessandro.deluca@unina.it \\ Christophe Reutenauer \\ Laboratoire de combinatoire et d'informatique mathématique \\ Université du Québec à Montréal \\ Case postale 8888, succursale Centre-ville \\ Montréal (Québec) H3C 3P8, Canada \\ reutenauer.christophe@uqam.ca \\ À l'ami Doron
}

Submitted: Dec 9, 2010; Accepted: Feb 8, 2011; Published: Oct 17, 2011

Mathematics Subject Classification: 68R15

\begin{abstract}
In this note we present some results on the Calkin-Wilf tree of irreducible fractions, giving an insight on the duality relating it to the Stern-Brocot tree, and proving noncommutative versions of known results relating labels of the CalkinWilf trees to hyperbinary expansions of positive integers. The main tool is the Christoffel tree introduced in a paper by Berstel and de Luca.
\end{abstract}

\section{Introduction}

The Stern-Brocot tree is a well-known infinite binary tree containing all positive rational numbers, or more precisely all irreducible fractions. It has been studied for decades because of its many useful properties which span topics like number theory, combinatorics and theoretical computer science. For instance, it is deeply connected with continued fractions, and it is a binary search tree for the positive rational numbers.

A closely related tree (already considered in [5] under the name of Raney tree) was recently studied by Calkin and Wilf [9]. While lacking the binary search tree property, the so-called Calkin-Wilf tree is arguably easier to generate using a simple recurrence; its 
$n$th fraction in a breadth-first visit (i.e., root first, then its child nodes left to right, then their children and so on) is given by $b(n-1) / b(n)$, where the sequence $(b(n))_{n \geq 0}$ satisfies $b(0)=0, b(2 n+1)=b(n)$, and $b(2 n+2)=b(n)+b(n+1)$ for all $n \geq 0$. The Stern diatomic sequence $(b(n))_{n \geq 0}$ was first considered in [13]; $b(n)$ was characterized in [9] as the number of hyperbinary expansions of the integer $n$, i.e., the number of ways $n$ can be written as a sum of powers of 2 , where each power appears at most twice.

The duality between these two binary trees of fractions (see [5]) can be characterized in terms of continued fraction expansions, or simply as follows: a node in the Stern-Brocot tree is labeled by the irreducible fraction $p / q$ if and only if the corresponding node in the Calkin-Wilf tree is $p^{\prime} / q^{\prime}$, where $0<p^{\prime}, q^{\prime}<p+q$ and

$$
p p^{\prime} \equiv q q^{\prime} \equiv 1 \quad(\bmod p+q)
$$

That is, $p^{\prime}$ and $q^{\prime}$ are the multiplicative inverses, modulo $p+q$, of $p$ and $q$ respectively.

Following [5], both trees can be viewed as specializations of a tree formed by ordered pairs of binary words, the Christoffel tree. This allows to identify the above duality with a well-known involution on Christoffel words (see [7]), and to give non-commutative interpretations for the properties of the two fraction trees. In this note we mainly focus on the duality and the sequence $b(n)$.

In the remainder of this section, we set some notation and review basic definitions and known results on the Stern-Brocot and Calkin-Wilf trees. In Section 2 we consider Christoffel words and the Christoffel tree, proving that corresponding fractions $p / q, p^{\prime} / q^{\prime}$ in the Stern-Brocot and Calkin-Wilf tree satisfy (1). In Section 3 we establish noncommutative recurrences for the Christoffel trees, defining a sequence of Christoffel words $(f(n))_{n \geq 1}$ which corresponds to the previously mentioned $(b(n))_{n \geq 0}$; in Theorem 3.1 we show that $f(n)$ encodes the relative lengths of the (reversed) hyperbinary expansions of $n$, taken in inverse lexicographic order.

The authors thank Jacques Labelle for bringing the article [9] to their attention. The first author thanks LaCIM and UQAM for their generous support during his stay in 2009-2010.

\subsection{The Stern-Brocot tree}

The Stern-Brocot tree was introduced independently in $[13,8]$. It is a complete, infinite binary tree of positive irreducible fractions, having the binary search property: each fraction is larger than all its left descendants, and smaller than its right descendants. It can be constructed by iterating the mediant operation: if $p / q$ and $p^{\prime} / q^{\prime}$ are the two closest ancestors (above and) to the left and to the right of a node (one being its parent), then its label is $\left(p+p^{\prime}\right) /\left(q+q^{\prime}\right)$. In order for this to make sense, the left and right "ancestors" of the root are defined respectively as $0 / 1$ and $1 / 0$. For instance, the fraction $3 / 4$ is obtained as the mediant of $2 / 3$ (its parent) and $1 / 1$ (the closest ancestor above and to the right of $3 / 4)$.

Let $L$ and $R$ denote, respectively, left and right moves down the tree, so that every word over the alphabet $\{L, R\}$ represents exactly one node of the tree (the empty word 
Figure 1: The Stern-Brocot tree

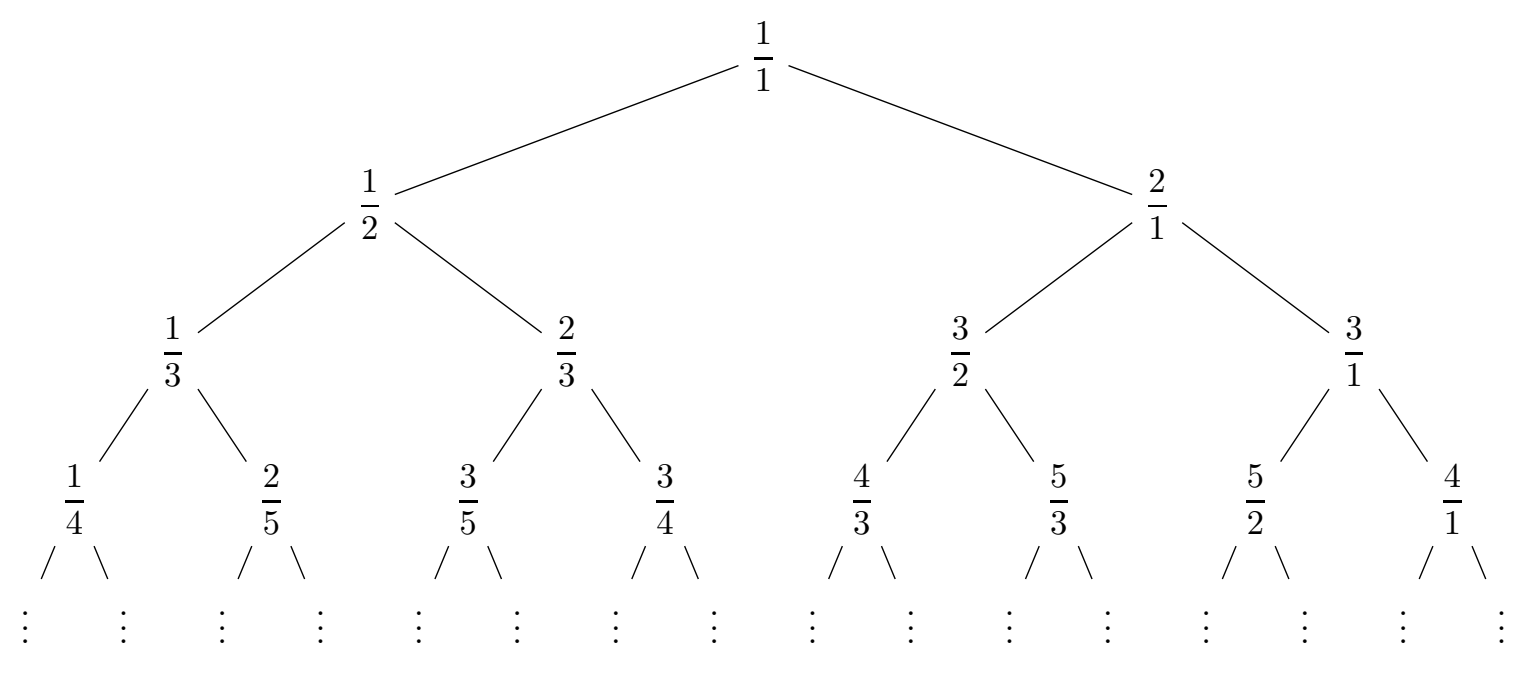

corresponding to the root). The following proposition shows that the Stern-Brocot tree is deeply related to continued fractions:

Proposition 1.1 (see $[11,5])$. Let $p / q$ be the label of a node in the Stern-Brocot tree which is represented by the word $R^{a_{0}} L^{a_{1}} \cdots R^{a_{n}}$, with $a_{0}, a_{n} \geq 0$ and $a_{i}>0$ for $0<i<n$. Then $p / q$ has the continued fraction expansion $\left[a_{0}, a_{1}, \ldots, a_{n}+1\right]$, i.e.

$$
\frac{p}{q}=a_{0}+\frac{1}{a_{1}+\frac{1}{\ddots \cdot+\frac{1}{a_{n}+1}}} .
$$

For more details about the Stern-Brocot tree, the reader is referred to [11].

\subsection{The Calkin-Wilf tree}

In [9], Calkin and Wilf examined a different binary tree comprising all irreducible fractions, generated by the following rules: the root is $1 / 1$, and every node $p / q$ has $\frac{p}{p+q}$ as its left child and $\frac{p+q}{q}$ as its right child. This tree had already been considered some years earlier by Berstel and de Luca [5], who named it the Raney tree in view of a paper by Raney [12]. We mention that natural $k$-ary generalizations of the Stern-Brocot and Raney trees, related to factors of episturmian words, were recently introduced by de Luca and Zamboni in [10].

Enumerating the fractions of the Calkin-Wilf tree, one easily notices that the denominator of any fraction appears to be equal to the numerator of the next one in a breadth-first visit. The following proposition, proved in [9], shows that this is actually true, thus summarizing two basic properties of the Calkin-Wilf tree: 
Figure 2: The Calkin-Wilf tree

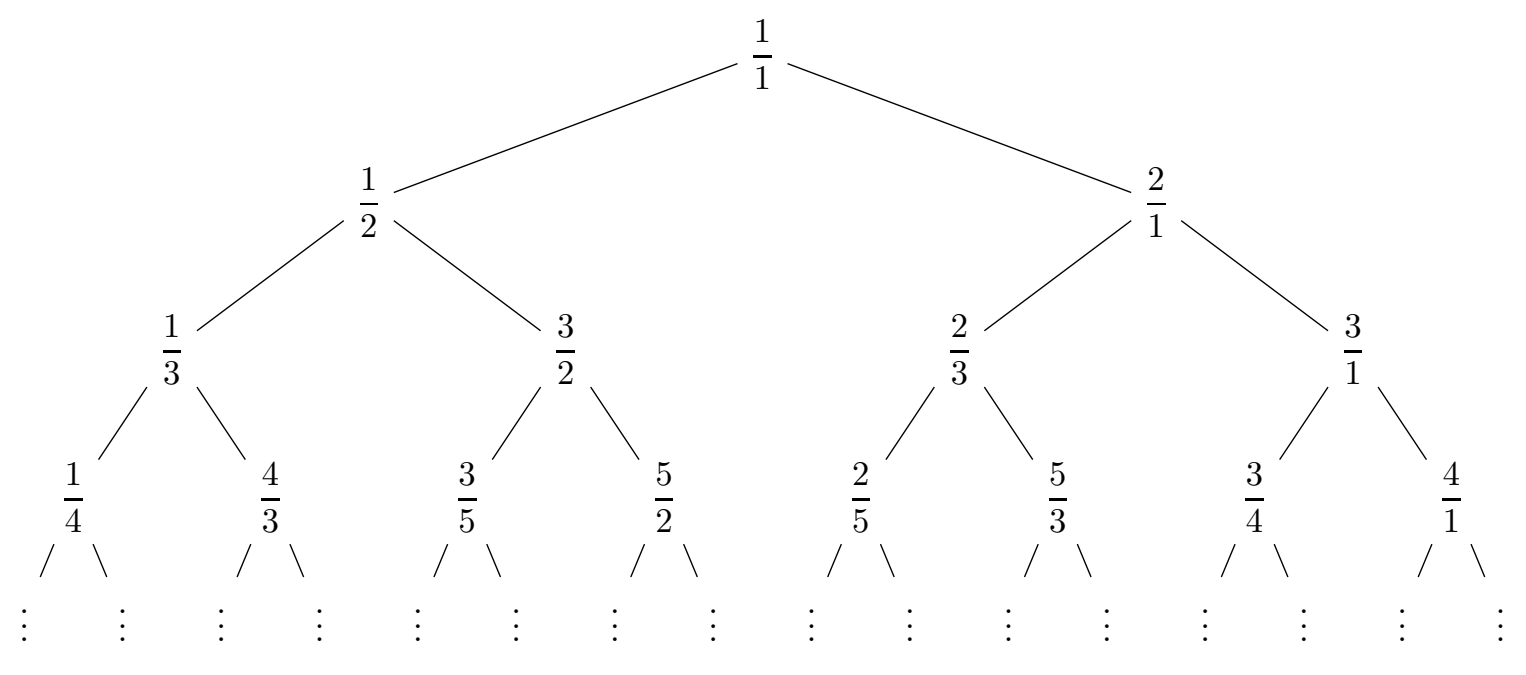

Proposition 1.2 ([9], see also [1]). Every positive irreducible fraction appears exactly once in the Calkin-Wilf tree, and there exists a sequence of integers $(b(n))_{n \geq 0}$ such that the $n$th fraction of the tree is given by $b(n-1) / b(n)$.

A hyperbinary expansion of the positive integer $n$ is a word $w=x_{1} x_{2} \cdots x_{k}$ with $x_{i} \in$ $\{0,1,2\}$ for $1 \leq i \leq k$, such that $n=\sum_{i=1}^{k} x_{i} 2^{k-i}$. Hence the usual binary representation of $n$ is a hyperbinary expansion (the only one not containing the digit 2). As a less trivial example, the hyperbinary expansions of $n=14$ are: 1110, 1102, 1022, and 222 .

Proposition $1.3([9])$. The value of $b(n)$ satisfies the recurrence $b(2 n+1)=b(n), b(2 n+$ $2)=b(n)+b(n+1)$ for all $n \geq 0$, and gives the number of hyperbinary expansions of $n$.

The following table gives the first values of $b(n)$ :

\begin{tabular}{c|cccccccccccccccc}
$n$ & 0 & 1 & 2 & 3 & 4 & 5 & 6 & 7 & 8 & 9 & 10 & 11 & 12 & 13 & 14 & 15 \\
\hline$b(n)$ & 1 & 1 & 2 & 1 & 3 & 2 & 3 & 1 & 4 & 3 & 5 & 2 & 5 & 3 & 4 & 1
\end{tabular}

Following [6, Chapter 5] we define the map $\nu_{2}:\{0,1\}^{*} \rightarrow \mathbb{N}$ which interprets words as integers in base 2, i.e., if $w=a_{0} a_{1} \cdots a_{n}$ with $a_{i} \in\{0,1\}$ for $0 \leq i \leq n$, then $\nu_{2}(w)=\sum_{i=0}^{n} a_{i} 2^{n-i}$. We recall that given a semiring $R$, a function $f: \mathbb{N} \rightarrow R$ is said to be 2-regular over $R$ if the formal power series

$$
S_{f}=\sum_{w \in\{0,1\}^{*}} f\left(\nu_{2}(w)\right) w \in R\langle\langle 0,1\rangle\rangle
$$

is rational, that is, if there exist a positive integer $n$, a morphism $\mu:\{0,1\}^{*} \rightarrow R^{n \times n}$, and two matrices $\lambda \in R^{1 \times n}, \gamma \in R^{n \times 1}$ such that for all $w$ one has $f\left(\nu_{2}(w)\right)=\lambda \mu(w) \gamma$. The 
integer $n$, called dimension of the linear representation $(\lambda, \mu, \gamma)$, also gives the number of states of a weighted automaton recognizing the series $S_{f}$.

In [2], Allouche and Shallit defined regular sequences and proved (see also $[4,3]$ ) that the function $b$ considered above is regular over $\mathbb{Z}$. We now give a slightly different proof, establishing regularity over $\mathbb{N}$ and specifying the dimension.

Proposition 1.4. The function $b$ is 2-regular over $\mathbb{N}$, and has a linear representation of dimension 2.

Proof. First, note that the function $b(n)$ satisfies, for any $n \geq 0$ (assuming $b(-1)=0$ conventionally):

$$
\begin{aligned}
b(0) & =1 \\
b(2 n) & =b(n-1)+b(n) \\
b(2 n+1) & =b(n) .
\end{aligned}
$$

Let $c(n)=b(n-1)$ for $n \geq 0$. Recall that (see [6]) for a function $h: \mathbb{N} \rightarrow \mathbb{N}$, one defines $(0 \circ h)(n)=h(2 n)$ and $(1 \circ h)(n)=h(2 n+1)$. We have

$$
\begin{aligned}
& (0 \circ b)(n)=b(2 n)=c(n)+b(n) \\
& (1 \circ b)(n)=b(2 n+1)=b(n) \\
& (0 \circ c)(n)=c(2 n)=b(2 n-1)=b(2(n-1)+1)=b(n-1)=c(n), \\
& (1 \circ c)(n)=c(2 n+1)=b(2 n)=c(n)+b(n) .
\end{aligned}
$$

Thus $0 \circ b=b+c, 1 \circ b=b, 0 \circ c=c, 1 \circ c=b+c$. It follows that the series $S_{b}$ defined for any word $w \in\{0,1\}^{*}$ by $\left(S_{b}, w\right)=b\left(\nu_{2}(w)\right)$ is rational over the semiring $\mathbb{N}$ and has the representation $(\lambda, \mu, \gamma)$, with

$$
\begin{gathered}
\lambda=\left(\begin{array}{ll}
1 & 0
\end{array}\right), \quad \gamma=\left(\begin{array}{l}
1 \\
0
\end{array}\right), \text { and } \\
\mu(0)=\left(\begin{array}{ll}
1 & 0 \\
1 & 1
\end{array}\right), \quad \mu(1)=\left(\begin{array}{ll}
1 & 1 \\
0 & 1
\end{array}\right) .
\end{gathered}
$$

This concludes our proof.

Figure 3: A weighted automaton recognizing $S_{b}=\sum_{w} b\left(\nu_{2}(w)\right) w$

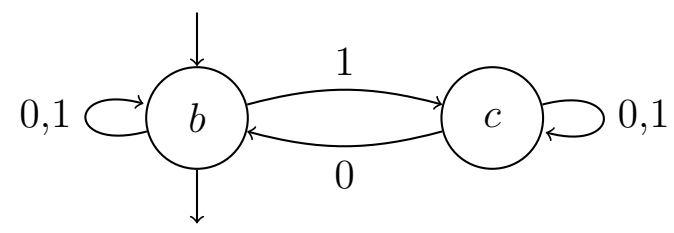




\section{The Christoffel tree}

Let us now consider a tree of ordered pairs of binary words, defined as follows: $(a, b)$ is the root, and every node $(u, v)$ has $(u, u v)$ and $(u v, u)$ as its left and right child, respectively (the product of two words being their concatenation). This tree was introduced in [5] as the Christoffel tree; its labels are exactly all Christoffel pairs, i.e., all pairs $(u, v)$ of Christoffel words such that $u<v$ in lexicographic order (with $a<b$ ) and $u v$ is again a Christoffel word. We recall (see also [7]) that the Christoffel word of slope $p / q$ over the alphabet $\{a, b\}$ ordered by $a<b$ is a word $w=x_{1} x_{2} \cdots x_{n}$ of length $n=p+q$, such that for $1 \leq i \leq n$ the letter $x_{i}$ is $a$ if

$$
\text { ip } \bmod n>(i-1) p \bmod n
$$

and $b$ otherwise. Every Christoffel word $w$ which is nontrivial (i.e., of length greater than 1) admits a unique decomposition $w=w_{1} w_{2}$ such that $w_{1}$ and $w_{2}$ are Christoffel words and $w_{1}<w_{2}$ in lexicographic order.

We also recall that the length of a word $w$ is denoted by $|w|$, whereas if $x$ is a letter, $|w|_{x}$ denotes the number of occurrences of the letter $x$ in $w$. Thus if $w$ is a word over the alphabet $\{a, b\}$, then $|w|=|w|_{a}+|w|_{b}$; furthermore, the slope of a Christoffel word $w$ is given by $|w|_{b} /|w|_{a}$.

Figure 4: The Christoffel tree

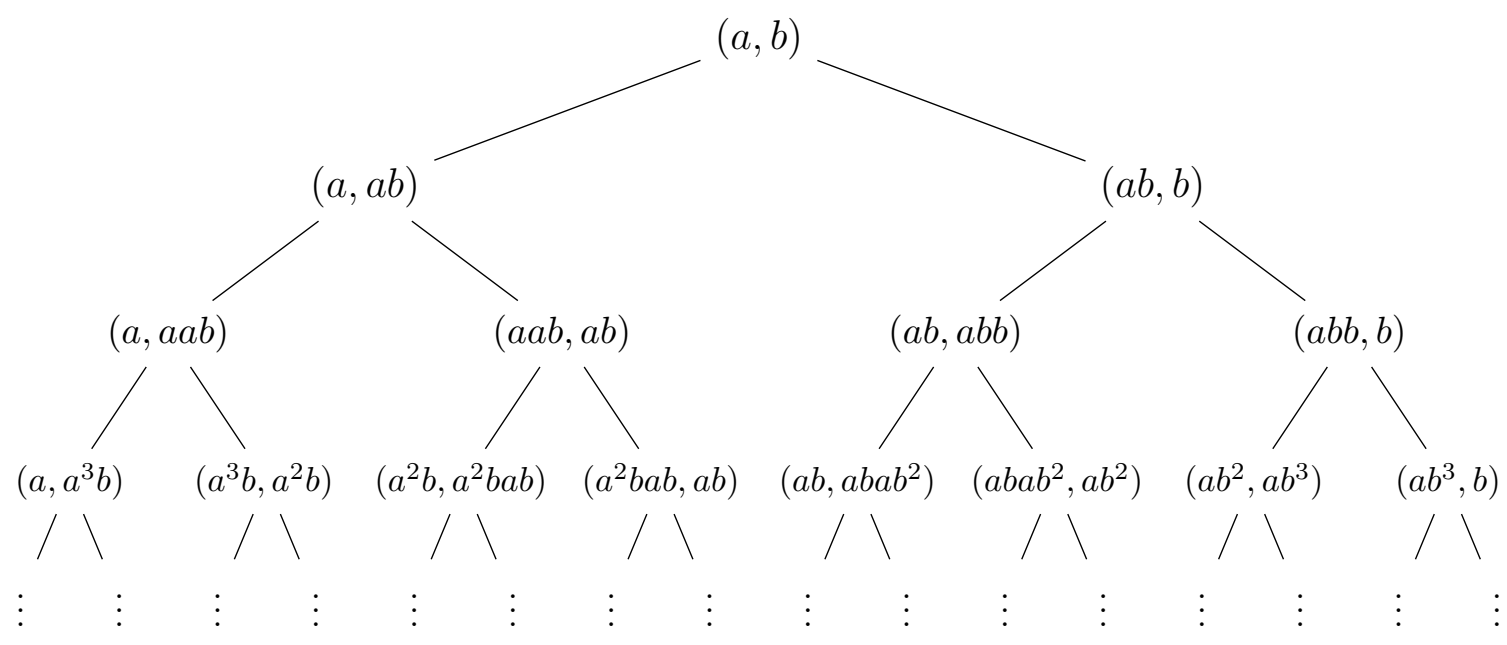

Note that the analogy with the Calkin-Wilf tree is almost perfect, in that for all nodes except for the rightmost ones of each level, the second term of the pair equals the first term of the next node to the right; however, the rightmost pair in the $k$ th level of the tree is $\left(a b^{k-1}, b\right)$ whereas the first pair in the next level is $\left(a, a^{k} b\right)$. Formally, we prove the following (cf. $[9,1]$ in the case of the Calkin-Wilf tree):

Lemma 2.1. If $(x, y)$ and $\left(x^{\prime}, y^{\prime}\right)$ are consecutive nodes on the same level of the Christoffel tree, then $y=x^{\prime}$. 
Proof. This is certainly true by definition if $(x, y)$ is a left child (in particular, it is true for the second node of the tree). Then suppose $(x, y)$ is a right child; its parent has to be $\left(x y^{-1}, y\right)$, whereas $\left(x^{\prime}, y^{\prime}\right)$ is the left child of $\left(x^{\prime}, x^{\prime-1} y^{\prime}\right)$. The two parents are also consecutive, so that by induction we get $y=x^{\prime}$ as desired.

We can still define a map $f(n)$, analogous to $b(n)$, giving the right term of the $n$th pair in the visit. This way, the $n$-th node in the Christoffel tree is $(a, f(n))$ if $n$ is a power of 2 (that is, if the node is on the leftmost branch), and $(f(n-1), f(n))$ otherwise, in view of the previous Lemma.

Theorem 2.2. The map $f$ can be defined by the following rules:

1. $f\left(2^{k}\right)=a^{k} b$ for all $k \geq 0$,

2. $f(2 n+1)=f(n)$,

3. $f(2 n)=f(n-1) f(n)$ if $n$ is not a power of 2.

Proof. The first equation describes the leftmost branch of the tree, whose nodes are of the form $\left(a, a^{k} b\right)$ as an immediate consequence of the definition.

The second equation describes right children, which have the same right term as their parent, again by definition.

Finally, the last equation describes left children of nodes that are not on the leftmost branch. Suppose this parent is the $n$-th node, so that its label is $(f(n-1), f(n))$ by induction. Then its left child is the $2 n$-th node, and by definition it is labeled by ( $f(n-$ $1), f(n-1) f(n))$. This proves the third equation and hence the theorem.

Along with Proposition 1.3, this implies that for all $n \geq 1,|f(n)|=b(n)$. The following table gives the first values of $f$ :

\begin{tabular}{c|cccccccccccc}
$n$ & 1 & 2 & 3 & 4 & 5 & 6 & 7 & 8 & 9 & 10 & 11 & 12 \\
\hline$f(n)$ & $b$ & $a b$ & $b$ & $a a b$ & $a b$ & $a b b$ & $b$ & $a a a b$ & $a a b$ & $a a b a b$ & $a b$ & $a b a b b$
\end{tabular}

The following basic theorem by Berstel and de Luca shows that the Calkin-Wilf and Stern-Brocot trees can be viewed as specializations of the Christoffel tree:

Theorem $2.3([5])$. Let $(u, v)$ be the label of a node in the Christoffel tree. Then $|u| /|v|$ is the corresponding node in the Calkin-Wilf (Raney) tree, and $|u v|_{b} /|u v|_{a}$ is the corresponding node in the Stern-Brocot tree.

Moreover, in [5] it is implicitly shown that the fraction obtained following a path (a word $W \in\{L, R\}^{*}$ ) on the Stern-Brocot tree is also obtained by following the reverse path $\tilde{W}$ on the Calkin-Wilf tree. In conjunction with Proposition 1.1, this gives:

Proposition 2.4 ([5]). Let $p / q$ be the label of a node in the Calkin-Wilf tree which is represented by the word $R^{a_{0}} L^{a_{1}} \cdots R^{a_{n}}$, with $a_{0}, a_{n} \geq 0$ and $a_{i}>0$ for $0<i<n$. Then $p / q$ has the continued fraction expansion $\left[a_{n}, a_{n-1}, \ldots, a_{0}+1\right]$. 
The two last results are examples of the duality occurring between the two trees of fractions, which can be seen as a consequence of the duality between Christoffel words which was explored in detail in [7]. For instance, consider the following lemma, proved in [7]:

Lemma 2.5. Let $w$ be a Christoffel word with $|w|>1$, and let $w=w_{1} w_{2}$ be its factorization in an increasing product of Christoffel words. Let $p / q$ be the slope of $w$, and let $p^{\prime}=\left|w_{1}\right|, q^{\prime}=\left|w_{2}\right|$. Then

$$
p p^{\prime} \equiv q q^{\prime} \equiv 1 \quad(\bmod |w|)
$$

Using Theorem 2.3 and the definition of the Christoffel tree, this implies the following

Corollary 2.6. Let $p / q$ be a fraction in the Stern-Brocot tree, and let $p^{\prime} / q^{\prime}$ be the label of the corresponding node in the Calkin-Wilf tree. Then $p+q=p^{\prime}+q^{\prime}$ and

$$
p p^{\prime} \equiv q q^{\prime} \equiv 1 \quad(\bmod p+q)
$$

This can also be inferred more directly from the following result, which is [7, Corollary $3.3]$ :

Proposition 2.7. Let $p / q$ and $p^{\prime} / q^{\prime}$ be two positive irreducible fractions. Then the paths leading to them in the Stern-Brocot tree are reverses of each other if and only if $p+q=$ $p^{\prime}+q^{\prime}$ and $p p^{\prime} \equiv q q^{\prime} \equiv 1(\bmod p+q)$.

\section{Main results}

Let us remark that the hyperbinary expansions of a natural number $n$ have just two possible lengths. Indeed, a hyperbinary expansion of $n$ cannot be longer than the usual binary representation of length $\ell=\left\lfloor\log _{2} n\right\rfloor+1$; furthermore, $n$ cannot have a representation of length $\ell-2$, since the greatest integer having such an expansion is $2 \cdot\left(2^{\ell-2}-1\right)=$ $2^{\ell-1}-2 \leq n-2$.

Thus we can naturally classify hyperbinary expansions of $n$ as long or short, respectively of length $\ell$ and $\ell-1$.

Theorem 3.1. Let $n>0$ be an integer. Then $f(n)$ is the word obtained by sorting the reversed hyperbinary expansions of $n$ in reverse lexicographic order, and then mapping each short (resp., long) expansion to the letter a (resp., b).

Proof. If $n=2^{k}$ for some $k \geq 0$, then $n$ has $b(n)=k+1$ hyperbinary expansions. The only long one is the binary expansion, $1 \underbrace{0 \cdots 0}_{k}$. The other $k$ short expansions are of the form

$$
\underbrace{1 \cdots 1}_{i} 2 \underbrace{0 \cdots 0}_{k-1-i}
$$


for $0 \leq i<k$. Among the reversals, the long one is also the smallest in lexicographic order (hence the greatest in reverse lex order), so that by mapping short expansions to $a$ and the long one to $b$, we get the word $a^{k} b=f(n)$.

We now prove the statement by induction on $n$. If $n=2 m+1$, then each hyperbinary expansion of $n$ can be written as $w 1$, where $w$ is an expansion of $m<n$. Trivially, adding a 1 to the left of all reversed expansions of $m$ does not change their order, nor their type (long or short). Hence the word obtained is the same, which by induction is $f(m)=f(n)$.

If $n=2 m$ and $m$ is not a power of 2 , then the hyperbinary expansions of $n$ are either of the form $w 0$ with $w$ an expansion of $m$, or of the form $w^{\prime} 2$ with $w^{\prime}$ an expansion of $m-1$. Clearly the reversals of the latter come before the ones of the former, in the inverse lexicographic order; moreover, as adding a 2 or a 0 to the left does not change the relative lengths of the expansions, nor their lexicographic ordering, we obtain the word $f(m-1) f(m)=f(n)$.

Example. The reversed expansions of $n=20$, in reverse lexicographic order, are:

\begin{tabular}{l|l} 
expansion & type \\
\hline 2121 & $a$ (short) \\
2102 & $a$ \\
21001 & $b$ (long) \\
0221 & $a$ \\
0202 & $a$ \\
02001 & $b$ \\
0012 & $a$ \\
00101 & $b$
\end{tabular}

and we have $f(20)=a a b a a b a b$.

We conclude with a few remarks. The restriction of our function $f$ gives a bijection between even positive integers and Christoffel words of length greater than 1. Indeed, as already observed in Section 2, the labels of the Christoffel tree are exactly all distinct Christoffel pairs, and every nontrivial Christoffel word is the product of the two components of exactly one Christoffel pair; this shows the bijection between nontrivial Christoffel words and nodes of the Christoffel tree, which in turn are in a bijection with their left children (even indexed nodes).

The equation $f(2 n)=f(n-1) f(n)$ gives the standard factorization of a nontrivial Christoffel word in an increasing product of two Christoffel words, and the proof of our last theorem gives a further interpretation of this factorization: the first factor $f(n-1)$ comes from hyperbinary expansions of $2 n$ ending in 2 , and the second factor $f(n)$ is given by the expansions ending in 0 .

\section{References}

[1] M. Aigner, G. M. Ziegler, Proofs from The Book, 3rd ed., Springer-Verlag, Berlin, 2004. 
[2] J.-P. Allouche, J. Shallit, The ring of $k$-regular sequences, Theoretical Comput. Sci. 98 (1992) 163-197.

[3] J.-P. Allouche, J. Shallit, Automatic Sequences, Cambridge University Press, Cambridge UK, 2003.

[4] J.-P. Allouche, J. Shallit, The ring of $k$-regular sequences, II, Theoretical Comput. Sci. 307 (2003) 3-29.

[5] J. Berstel, A. de Luca, Sturmian words, Lyndon words and trees, Theoretical Comput. Sci. 178 (1997) 171-203.

[6] J. Berstel, C. Reutenauer, Noncommutative Rational Series With Applications, vol. 137 of Encyclopedia of Mathematics and its Applications, Cambridge University Press, Cambridge UK, 2010.

[7] V. Berthé, A. de Luca, C. Reutenauer, On an involution of Christoffel words and Sturmian morphisms, European J. Combin. 29 (2008) 535-553.

[8] A. Brocot, Calcul des rouages par approximation, nouvelle méthode, Revue Chronométrique 3 (1861) 186-194.

[9] N. Calkin, H. S. Wilf, Recounting the rationals, Amer. Math. Monthly 107 (2000) 360-363.

[10] A. de Luca, L. Q. Zamboni, Involutions of epicentral words, European J. Combin. 31 (2010) 867-886.

[11] R. L. Graham, D. E. Knuth, O. Patashnik, Concrete mathematics, 2nd ed., AddisonWesley Publishing Company, Reading, MA, 1994.

[12] G. N. Raney, On continued fractions and finite automata, Mathematische Annalen 206 (1973) 265-283.

[13] M. A. Stern, Über eine zahlentheoretische Funktion, J. Reine Angew. Math. 55 (1858) $193-220$. 